\title{
INTERACTIONS OF AN OSCILLATING RECTANGULAR MEMBRANE WITH A COMPRESSIBLE FLUID
}

\author{
MANUEL GASCÓN-PÉREZ \\ Escuela Técnica Superior de Ingeniería Aeronáutica y del Espacio, UPM, \\ 28040 Madrid, Spain \\ manuel.gascon@upm.es
}

Received

Revised

\begin{abstract}
Interaction of an oscillating membrane with a fluid is important because the wide variety of technological applications. A boundary element method has been employed for the analysis of a vibrating rectangular membrane in contact with a compressible fluid at rest. The deformation modes of themembrane correspond to the vacuum case. For the calculation of the pressure jump over the membrane the Rayleigh's int egral equation for the fluid pressure is employed taking into account the fluid-membrane interface boundary condition. Considering the membrane equation and applying a collocation method, the natural frequencies of the interacting system are obtained. The influence of various parameters such as aspect ratio, fluid density and membrane dimension, on these frequencies are evaluated. Furthermore, the influence of the wave number on the fluid mass coefficient and the acoustic damping ratio are evaluated. The validity of the method is deduced when comparing the results obtained by other authors and theories.
\end{abstract}

Keywords: rectangular membrane; fluid-structure; natural frequencies; boundary element method.

\section{Introduction}

The influence of a fluid on the dynamic characteristics of a vibrating membrane has been studied for many years motivated by the solutions of engineering problems, see Jenkins et al. [2006].These structures, with no flexural stiffness, are found in various applications, for example, architectural and civil structures, diaphragms in switches and sensors, biological prosthes is such as aortic valves , and satellite-based applications such as solar arrays, inflatable antennas and reflectors. Other applications of devices incorporating resonating membranes that are frequently used in MicroElectro-Mechanical Systems MEMS are: thermo-pneumatic membrane actuators, see Jeong et al [2000], flow control valves, see Grosjean et al [1999], valveless micro pumps, see Pan et al [2003], micro ultrasonic transducers, see Caronti et al [2002], and pressure sensors, see Defay et al [2002].

The performance efficiency of these reflecting surfaces in space-based applications depends not only on the geometrical accuracy of the surface but also on their vibration characteristics, which are considerably affected by the surrounding medium due to their lightness. It is important then, in the development of these light and large structures, to have a method that estimates the effect that the surrounding fluid will have on the natural frequencies of the structure. In this way it can be avoided by testing the structure in a vacuum chamber, which for a large antenna or panel can be difficult and costly.

Investigation of the fluid-membrane or fluid-plate interaction problem has been done by many researchers. Kwak [1996 ] studied the hydro-elastic vibration of a rectangular plate concerned 


\section{M. Gascón-Pérez}

with the virtual mass effect on the natural frequencies and mode shapes of rectangular plates due to the presence of water on one side of the plate. Berry et al [1990] considered a general formulation for the sound radiation from rectangular baffled plates with arbitrary boundary conditions. Kerboua et al. [2008] analyzed the vibration of rectangular plates completely submerged or floating on the free surface of fluid. Crighton [1983] investigated the response of a fluid loaded membrane under a concentrated excitation force by the Green`s function. Erchiqui et al. [2015] who made a classification study of high strained membranes under loads of fluid pressure. The work of Gascón-Pérez [2017] where the vibroacoustic behavior of a membrane drum is analyzed by the application of a boundary element method.

The vibration of a rectangular membrane clamped along its edges and fully submerged in an infinite fluid (compressible or incompressible) is analyzed in this paper. Applying a boundary element method, taking into account the Rayleigh`s integral formulation for the fluid pressure and the equation of motion of the membrane, the frequencies of the coupled system are evaluated by a collocation method leading to an eigenvalue problem.

Finally the effects of fluid parameters such as density, and the membrane parameters such as membrane dimension and aspect ratio, on the free vibration response of the coupled system are investigated, as well as the variation of the fluid mass coefficient and the acoustic damping ratio with the wave number.

\section{Problem Formulation}

The rectangular membrane immersed in the fluid satisfies the equation, see Wang et al. [2014]:

$$
T \Delta w(x, y, t)-\rho_{m} t_{h} \frac{\partial^{2} w}{\partial t^{2}}=\Delta p(x, y, t)
$$

Where $T$ is the membrane tension, $t_{h}$ the membrane thickness, and $\rho_{m}$ the material density of the membrane.

Fig.1 is represented by a rectangular membrane submerged in an infinite fluid and the characteristic dimensions that define it.

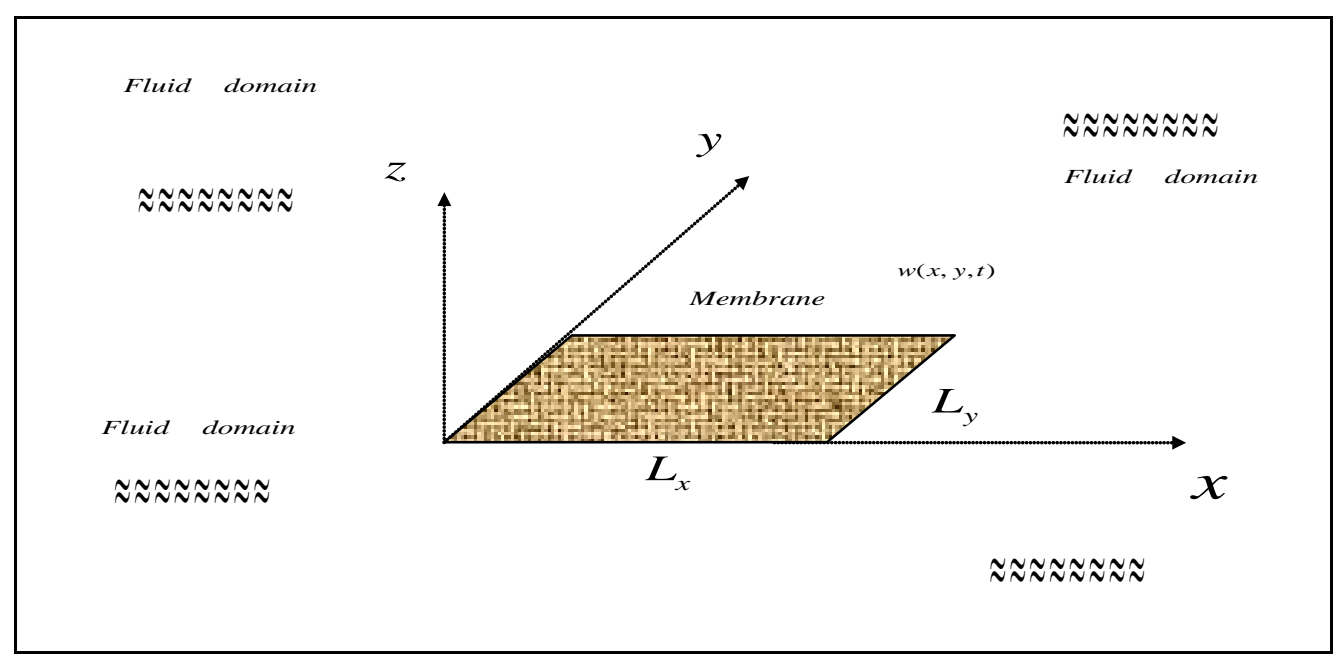

Fig. 1. Scheme of a rectangular membrane submerged in fluid, showing the different geometric parameters

It is assumed that the only action over the membrane is the fluid pressure jump $\Delta p$ between its upper and lower sides. 
The pressure field is obtained by solving the wave equation on the fluid domain that is supposed at rest

$$
\frac{1}{a_{\infty}^{2}} \frac{\partial^{2} p}{\partial t^{2}}-\Delta p=0
$$

If the momentum equation applied at the surface of the membrane is considered, the following boundary condition is obtained:

$$
\frac{\partial p}{\partial z}=-\rho_{\infty} \frac{\partial^{2} w}{\partial t^{2}} \text { at } z= \pm 0 \quad \text { and } 0<x<L_{x}, \quad 0<y<L_{y}
$$

The membrane placed at the $z=0$ plane, and $L_{x}, L_{y}$ are the side lengths of the membrane.

If it is assumed that the motion is harmonic, the response of both the fluid and structure is of the form:

$$
w(x, y, t)=\tilde{w}(x, y) \cdot e^{-i \omega t} \text { and } p(x, y, z, t)=\tilde{p}(x, y, z) \cdot e^{-i \omega t}
$$

The deformation function of the membrane, when interacting with the fluid can be expanded as a linear combination of the normalized modes of the membrane in vacuum:

$$
\tilde{w}(x, y)=\sum_{m} \sum_{n} W_{m}^{n}(x, y) \cdot q_{m}^{n}
$$

Where $q_{m}^{n}$ represent the weight coefficient associated to the deformation mode $W_{m}^{n}$ of the membrane.

The functions $W_{m}^{n}(x, y)$ for a membrane clamped along its edges, have the general expression Wang [2014]

$$
W_{m}^{n}(x, y)=\sin \left(\frac{m \pi x}{L_{x}}\right) \cdot \sin \left(\frac{n \pi y}{L_{y}}\right)
$$

The indexes $m, n$ represent the number of nodal lines in the $y, x$ directions respectively.

Considering the wave equation for the fluid pressure, equation (2.2) and the assumption of harmonic motion, equation (2.4), it is deduced:

$$
\Delta \tilde{p}+k^{2} \tilde{p}=0
$$

Where $k=\frac{\omega}{a_{\infty}}$ is the wave number.

Taking into account the boundary condition, equation (2.3), and if equations (2.4) and (2.5) are substituted, it is obtained:

$$
\begin{aligned}
& \left.\frac{\partial \tilde{p}(x, y, z)}{\partial z}\right|_{z=0}=\rho_{\infty} \omega^{2} \tilde{w}(x, y)=\rho_{\infty} \omega^{2} \sum_{n} \sum_{m} W_{m}^{n}(x, y) \cdot q_{m}^{n} \\
& \text { at } z=0 \text { and } 0<x<L_{x}, \quad 0<y<L_{y}
\end{aligned}
$$

Then the pressure distribution can also be expressed as a linear combination of the pressure modes:

$$
\tilde{p}(x, y, z)=\sum_{m} \sum_{n} P_{m}^{n}(x, y, z)
$$

and now equation (2.8) can be expressed as:

$$
\left.\frac{\partial P_{m}^{n}}{\partial z}\right|_{z=0}=\rho_{\infty} \omega^{2} W_{m}^{n}(x, y) q_{m}^{n} \quad 0<x<L_{x}, \quad 0<y<L_{y}
$$

Taking into account equations (2.9) and (2.7), the equation for the pressure mode $P_{m}^{n}$ verifies:

$$
\frac{\partial^{2} P_{m}^{n}}{\partial x^{2}}+\frac{\partial^{2} P_{m}^{n}}{\partial y^{2}}+\frac{\partial^{2} P_{m}^{n}}{\partial z^{2}}+k^{2} P_{m}^{n}=0
$$




\section{Calculus of the pressure jump over the membrane}

Considering the infinite fluid boundary and the membrane boundary, see Fig.1, and applying the Rayleigh`s integral equation, the following expression is obtained for the pressure mode (from Refs. Crocker [2007] and Fahy [2001] ):

$$
P_{m}^{n}(x, y, z)=-\frac{1}{4 \pi} \iint_{S_{m}} \Delta P_{m}^{n}(\xi, \eta) \frac{\partial}{\partial z}\left(\frac{e^{i k \sqrt{(x-\xi)^{2}+(y-\eta)+z^{2}}}}{\sqrt{(x-\xi)^{2}+(y-\eta)+z^{2}}}\right) d \xi d \eta
$$

With $x, y$ and $\xi, \eta$ the Cartesian coordinates of the field and source points, $S_{m}$ the membrane surface, and $\Delta P_{m}^{n}$ the pressure jump over the membrane.

Applying the boundary condition, equation (2.10), is obtained

$$
\rho_{\infty} \omega^{2} W_{m}^{n}(x, y) q_{m}^{n}=-\frac{1}{4 \pi} \iint_{S_{m}} \Delta P_{m}^{n}(\xi, \eta) \frac{\partial^{2}}{\partial z^{2}}\left(\frac{e^{i k \sqrt{(x-\xi)^{2}+(y-\eta)+z^{2}}}}{\sqrt{(x-\xi)^{2}+(y-\eta)+z^{2}}}\right)_{z=0} d \xi d \eta
$$

Let

$$
\Psi(x-\xi, y-\eta, z, k)=\left.\frac{\partial^{2}}{\partial z^{2}}\left(\frac{e^{i k \sqrt{(x-\xi)^{2}+(y-\eta)^{2}+z^{2}}}}{\sqrt{(x-\xi)^{2}+(y-\eta)^{2}+z^{2}}}\right)\right|_{z=0}
$$

be the kernel of the integral equation. The solution to equation (2.13) will be obtained approximately by applying a collocation method.

The membrane is divided into $N \times N$ rectangular panels. At the center of each panel or the collocation points whose coordinates are $\left(x_{i}, y_{j}\right)$ or $\left(\bar{\xi}_{r}, \bar{\eta}_{s}\right)$, the equation (2.13) is fulfilled, where $\bar{\xi}_{r}=\frac{\xi_{r+1}+\xi_{r}}{2}$ and $\bar{\eta}_{s}=\frac{\eta_{s+1}+\eta_{s}}{2}$

Then this equation can be expressed for the collocation method:

$$
\rho_{\infty} \omega^{2} W_{m}^{n}\left(x_{i}, y_{j}\right) q_{m}^{n}=-\frac{1}{4 \pi} \sum_{r=1}^{N} \sum_{s=1}^{N} \Delta P_{m}^{n}\left(\bar{\xi}_{r}, \bar{\eta}_{s}\right) \int_{\xi_{r}}^{\xi_{r+1}} \int_{\eta_{s}}^{\eta_{s+1}} \Psi\left(x_{i}-\xi, y_{i}-\eta, 0, k\right) d \xi d \eta
$$

So finally we end up with a linear system of algebraic equations to determine the pressure jump of the mode at each panel $\Delta P_{m}^{n}\left(\bar{\xi}_{r}, \bar{\eta}_{s}\right)$.

When evaluating the integral

$$
\Omega_{i j}^{r s}=-\frac{1}{4 \pi} \int_{\xi_{r}}^{\xi_{r+1}} \int_{\eta_{s}}^{\eta_{s+1}} \Psi\left(x_{i}-\xi, y_{i}-\eta, 0, k\right) d \xi d \eta
$$

special care must be taken of the singularity when $\xi \rightarrow x_{i}$ and $\eta \rightarrow y_{i}$. To avoid this singularity, the Kernel function is split into a regular part and a singular part, see Gascón-Pérez et al. [2015].

$$
\Psi=\Psi_{R}+\Psi_{S}
$$

The singular part is integrated analytically and the regular part is integrated numerically.

The singular part is

$$
\Psi_{S}=-\frac{1}{\Theta^{3}}-\frac{k^{2}}{2 \Theta}
$$

Where $\Theta=\sqrt{(x-\xi)^{2}+(y-\eta)^{2}+z^{2}}$ and $\Psi_{R}=\Psi-\Psi_{S}$

Where $\Omega_{i j S}^{r s}$ is the value of the singular integral

$$
\Omega_{i j S}^{r s}=\frac{1}{4 \pi} \int_{\xi_{r}}^{\xi_{r+1}} \int_{\eta_{s}}^{\eta_{s+1}}\left(\frac{1}{\Theta^{3}}+\frac{k^{2}}{2 \Theta}\right) d \xi d \eta
$$


Taking its principal value, from Roussos [2014], the following is obtained:

$$
\Omega_{i j S}^{r s}=\frac{1}{\pi}\left\{\begin{array}{l}
-\frac{\sqrt{(\delta x / 2)^{2}+(\delta y / 2)^{2}}}{(\delta x \cdot \delta y / 4)}+ \\
+\frac{k^{2}}{2} \cdot\left[\begin{array}{l}
(\delta y / 2) \ln \left(\frac{(\delta x / 2)+\sqrt{(\delta x / 2)^{2}+(\delta y / 2)^{2}}}{(\delta y / 2)}\right)+ \\
+(\delta x / 2) \ln \left(\frac{(\delta y / 2)+\sqrt{(\delta x / 2)^{2}+(\delta y / 2)^{2}}}{(\delta x / 2)}\right)
\end{array}\right]
\end{array}\right\}
$$

Where $\delta x$ and $\delta y$ are the magnitude of the sides of each rectangular panel.

Thus finally the system of equations (2.15) is expressed as:

$$
\rho_{\infty} \omega^{2} W_{m}^{n}\left(x_{i}, y_{j}\right) q_{m}^{n}=\sum_{r=1}^{N} \sum_{s=1}^{N}\left(\Omega_{i j R}^{r s}+\Omega_{i j S}^{r s}\right) \Delta P_{m}^{n}\left(\bar{\xi}_{r}, \bar{\eta}_{s}\right)
$$

Where $\Omega_{i j R}^{r s}$ is the numerical integration of $\Psi_{R}$

In the matrix form this system of equations (2.21) can be expressed as:

$$
\left[\Omega_{i j}^{r s}\right]\left\{\Delta P_{m}^{n}\left(\bar{\xi}_{r}, \bar{\eta}_{s}\right)\right\}=\rho_{\infty} \omega^{2}\left\{W_{m}^{n}\left(x_{i}, y_{j}\right)\right\} q_{m}^{n}
$$

So finally the modal pressure jump is given by

$$
\left\{\Delta P_{m}^{n}\left(\bar{\xi}_{r}, \bar{\eta}_{s}\right)\right\}=\rho_{\infty} \omega^{2}\left[\Omega_{i j}^{r s}\right]^{-1}\left\{W_{m}^{n}\left(x_{i}, y_{j}\right)\right\} q_{m}^{n}
$$

For solving the equation (2.1), the equations (2.4), (2.5) and (2.9) are considered and it is obtained:

$$
T \nabla^{2} W_{m}^{n}(x, y) q_{m}^{n}+\rho_{m} t_{h} \omega^{2} W_{m}^{n}(x, y) q_{m}^{n}=\Delta P_{m}^{n}(x, y)
$$

Where $\nabla^{2}$ is a differential operator of the form $\nabla^{2}=\left(\frac{\partial^{2}}{\partial x^{2}}+\frac{\partial^{2}}{\partial y^{2}}\right)$.

By multiplying equation (2.24) by the mode $W_{u}^{v}$, and integration over the surface of the membrane, the following equation is obtained:

$$
\iint_{S_{m}} T \Delta W_{m}^{n} W_{u}^{v} q_{m}^{n} d S+\rho_{m} t_{h} \omega^{2} \iint_{S_{m}} W_{m}^{n} W_{u}^{v} q_{m}^{n} d S=\iint_{S_{m}} \Delta P_{m}^{n} W_{u}^{v} d S
$$

Applying a collocation method and considering the expression (2.23) for the pressure jump over the membrane, the following scalar equation is obtained:

$$
\begin{aligned}
& T\left\lfloor\Delta W_{m}^{n}\right\rfloor[\Delta S]\left\{W_{u}^{v}\right\} q_{m}^{n}+\rho_{m} t_{h} \omega^{2}\left\lfloor W_{m}^{n}\right\rfloor[\Delta S]\left\{W_{u}^{v}\right\} q_{m}^{n}= \\
& =\rho_{\infty} \omega^{2}\left\lfloor W_{m}^{n}\right\rfloor\left[[\Omega]^{-1}\right]^{T}[\Delta S]\left\{W_{u}^{v}\right\} q_{m}^{n}
\end{aligned}
$$

Making variation of $m, n, u, v=0,1,2, \ldots \ldots \ldots ., N$ the following systemis obtained, leading to an eigenvalue problem:

$$
\left[[K]-\omega^{2}\left([M]+\left[M_{F}\right]\right)\right]\{q\}=\{0\}
$$

Where the coefficients of these matrices are expressed:

$$
\begin{gathered}
K_{m u}^{n v}=T\left\lfloor\nabla^{2} W_{m}^{n} \mid[\Delta S]\left\{W_{u}^{v}\right\}\right. \\
M_{m u}^{n v}=\rho_{m} t_{h}\left|W_{m}^{n}\right|[\Delta S]\left\{W_{u}^{v}\right\} \\
M_{F m u}^{n v}=\rho_{\infty}\left\lfloor W_{m}^{n}\right\rfloor\left[[\Omega]^{-1}\right]^{T}[\Delta S]\left\{W_{u}^{v}\right\}
\end{gathered}
$$

Where $[\Delta S]$ is a matrix containing the surface area of each rectangular panel. 
It must be said that the fluid matrix $\left[M_{F}\right]$ for compressible cases is a complex matrix and its coefficients are functions of the wave number $k=\frac{\omega}{a_{\infty}}$. For the solution of this nonlinear eigenvalue problem, equation (2.27), an iteration procedure is used to obtain the natural frequencies. These frequencies are obtained one by one, since the wave number depends on the frequency, while for an incompressible fluid $k=0$, all of them are obtained at the same time.

The iteration procedure converges in two or three steps. Once the natural frequencies of the coupled fluid-structure are determined, the normal wet modes can be computed by determining the eigenvector $\left\{q_{i}\right\}$ and expressed as a linear combination of the normal modes of the structure in vacuum.

\section{Results}

For verification of the method, in Table 1, the frequencies of a simply supported rectangular plate in contact with fluid and in vacuum, $f_{l m}^{n}$ and $f_{v m}^{n}$, are compared with the results of Kwak [1996] that analyzes the Navmi factor of a rectangular plate, calculating the change in the natural frequencies of a thin rectangular plate in an aperture of an infinite rigid plane wall in contact with water (incompressible fluid). For this case, the whole procedure is similar to that of the membrane and the equation (2.1) is substituted by the equation of motion of the plate, from Wang et al. [2014]:

$$
D \nabla^{4} w(x, y, t)+\rho_{m} t_{h} \frac{\partial^{2} w}{\partial t^{2}}=\Delta p(x, y, t)
$$

With $D=\frac{E \cdot t_{h}{ }^{3}}{12\left(1-v^{2}\right)}$ the flexural rigidity, $E$ the elasticity modulus of the material, $t_{h}$ the plate thickness, $v$ the Poisson modulus and $\rho_{m}$ the material density of the plate.

Moreover, in this case the functions $W_{m}^{n}$ are the modes of deformation of the plate that have the general expression, see Wang et al. [2014]: $W_{m}^{n}(x, y)=\sin \left(\frac{m \pi x}{L_{x}}\right) \cdot \sin \left(\frac{n \pi y}{L_{y}}\right)$

The frequency $f_{l m}^{n}$ is associated with the mode $W_{m}^{n}$ of the plate in contact with water, and the frequency $f_{v m}^{n}$ corresponding with the plate in the vacuum, both expressed in $\mathrm{Hz}$.

As it can be observed from Table 1, there is a slight difference in the results of the present method compared with that obtained by Kwak [1996], depending on the mode considered for the different values of nodal parameters $m$ and $n$, the validity of this method can be concluded.

It must be said that the case studied by Kwak is not exactly the same as the one considered here because the rectangular plate is situated in an aperture of an infinite rigid plane wall in contact with water and not fully filled submerged in water as in the present case. Furthermore, one can observe a great reduction in frequency in the wet case (with water) with respect to the vacuum case, and this reduction is higher, the lower the value of the mode considered, see Gascón-Pérez et al. [2015].

Table 1. Natural frequencies (Hz) for a simply supported rectangular steel plate (in vacuum and water) immersed in fluid, of side lengths $L_{x}=5 \mathrm{~m}, L_{y}=4 \mathrm{~m}$, and thickness $t_{h}=1 \mathrm{~cm}$

\begin{tabular}{|c|c|c|c|c|}
\hline$m, n$ & $f_{v}$ & $f_{l} \quad$ (Kwak) & $f_{l}$ (present method) & $\begin{array}{c}\text { Relative } \\
\text { discrepancy (\%) }\end{array}$ \\
\hline 1,1 & 2,498 & 0,398 & 0,435 & 8,5 \\
\hline
\end{tabular}




\begin{tabular}{|l|l|l|l|l|}
\hline 1,2 & 5,422 & 1,093 & 1,171 & 7,1 \\
\hline 2,1 & 7,067 & 1,561 & 1,623 & 3,9 \\
\hline 2,2 & 9,991 & 2,468 & 2,503 & 1,4 \\
\hline
\end{tabular}

The next results were obtained for the case of an aluminium rectangular membrane submerged in air (compressible fluid), and the following properties are considered:

Side lengths $L_{x}=0.5 \mathrm{~m}, L_{y}=0.4 \mathrm{~m}$, thickness $t_{h}=0.04 \mathrm{~mm}$, tension $T=100 \frac{\mathrm{N}}{\mathrm{m}}$,

material density $\rho_{m}=2700 \frac{\mathrm{kg}}{\mathrm{m}^{3}}$

Fig. 2 presents the frequency parameter $C_{\omega}$ defined as $C_{\omega}=\sqrt{\frac{\rho_{m} t_{h}}{T}} L_{x} \cdot \omega$, as a function of the length $L_{x}$ of the side of the rectangular membrane, for different nodal parameters $m, n$, taking into account that the ratio $\frac{L_{x}}{L_{y}}$ is maintained constant, where $L_{y}$ is the other side length. As it can be seen from the Figure, the frequency parameter decreases with the side length and the ratio of decrease is higher, the lower the values of the side length. Although for the vacuum case, the frequency parameter remains constant, and these values are represented in the same Figure.

Fig. 3 presents the frequency parameter $C_{\omega}$ as a function of the membrane aspect ratio $\frac{L_{y}}{L_{x}}$ in the range $[0.1-1]$, for different nodal parameters $m, n$. As it can be seen from the Figure below, the frequency parameter decreases with the aspect ratio and the rate of decrease is higher, the lower the values of $\frac{L_{y}}{L_{x}}$.

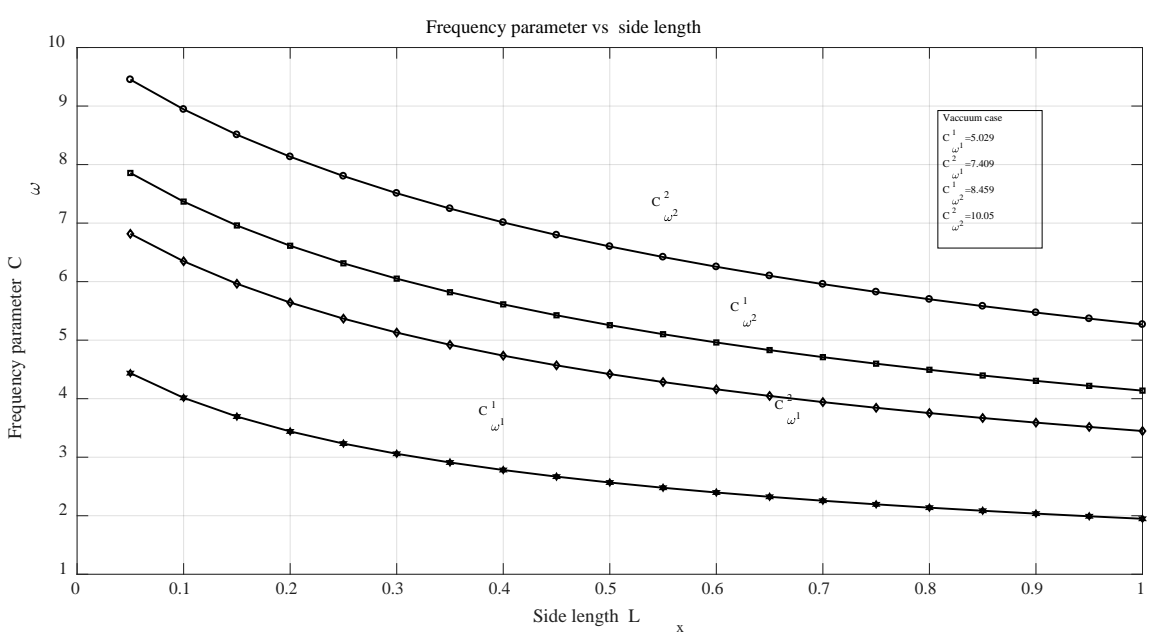

Fig. 2. Variation of membrane frequency parameter $C_{\omega}$ with fluid and in vacuum, with the length of the side of the membrane for different nodal parameters 


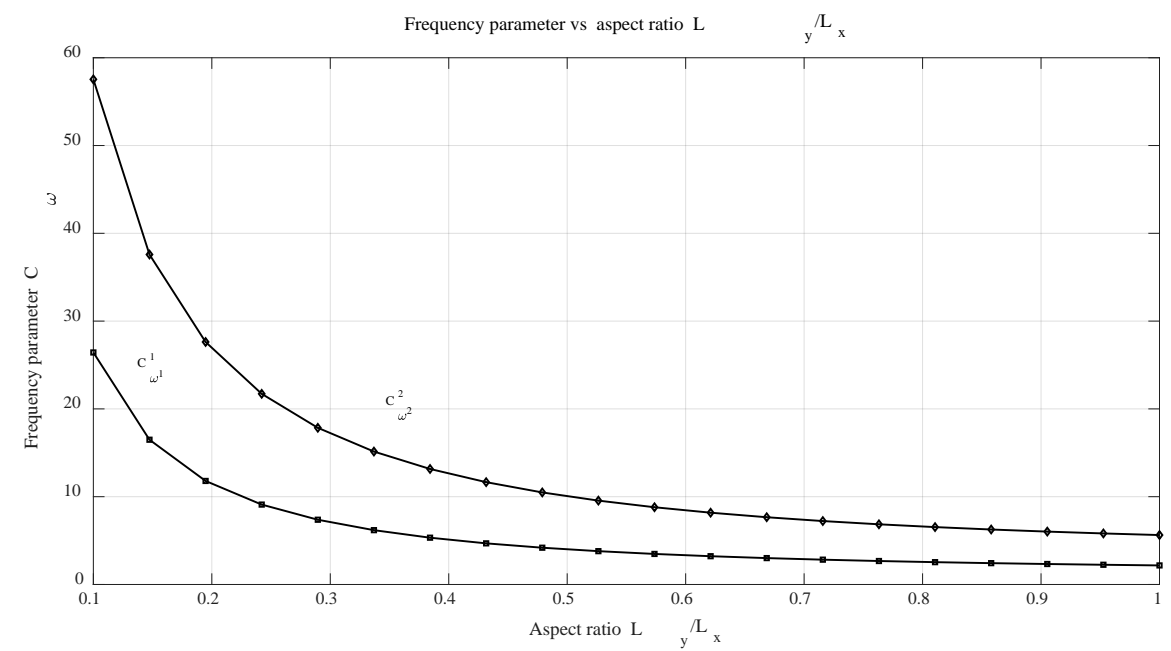

Fig. 3. Variation of membrane frequency parameter $C_{\omega}$ with fluid, with the membrane aspect ratio for different nodal parameters

Fig. 4 presents the frequency parameter $C_{\omega}$ as a function of the membrane aspect ratio $\frac{L_{y}}{L_{x}}$, but in this case, it shows the range [1 - 40] instead. As it can be seen from the Figure, the frequency parameter presents a minimum at an approximate value $L_{y} / L_{x}=6.5$ and for higher values of the aspect ratio tends to increase to an asymptotic value.

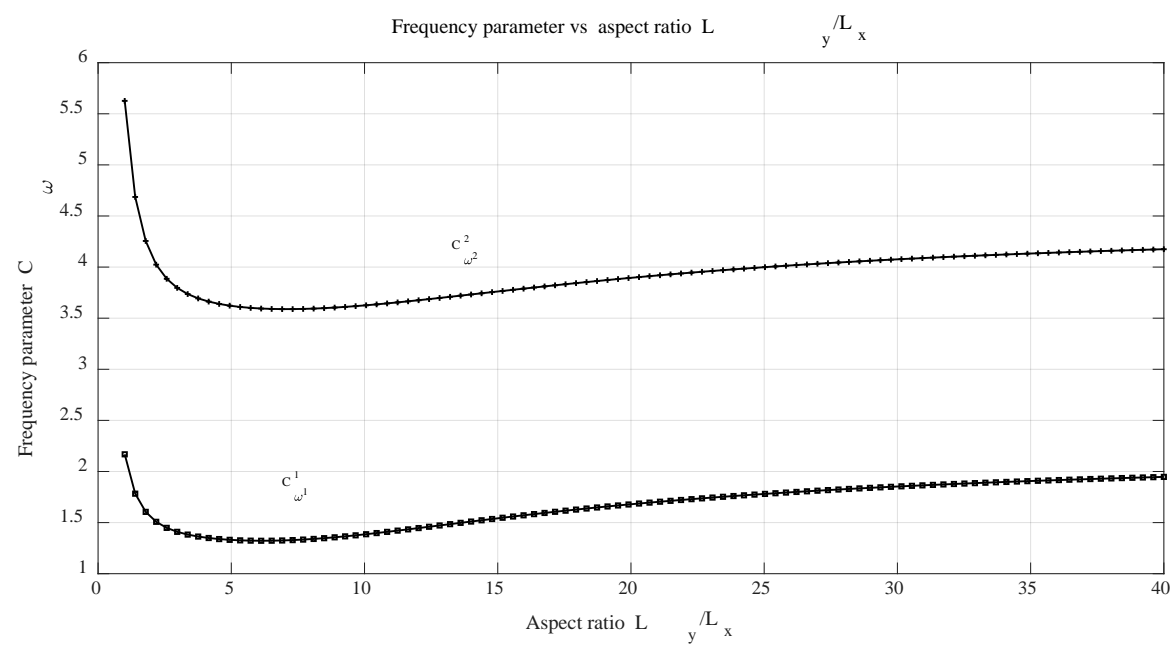

Fig. 4. Variation of membrane frequency parameter $C_{\omega}$ with fluid, with the membrane aspect ratio for different nodal parameters

Fig. 5 presents the fluid mass coefficient as function of the wave number $k=\frac{\omega}{a_{\infty}}$. This coefficient is defined as $C_{M f}=\frac{M_{f}}{4 \pi L_{x}^{2} L_{y} \rho_{\infty}}$ where $M_{f}$ is the diagonal term of the fluid mass matrix that contributes to the reduction of the frequency in relation to the vacuum value. $C_{M f}$ presents an oscillating variation with the wave number $k$, reaching a maximum for a certain 
value of $k$ and decreasing to a zero value for high values of $k$, so this fluid mass coefficient is analogous to a transfer function relating the fluid pressure jump and the deformation of the membrane.

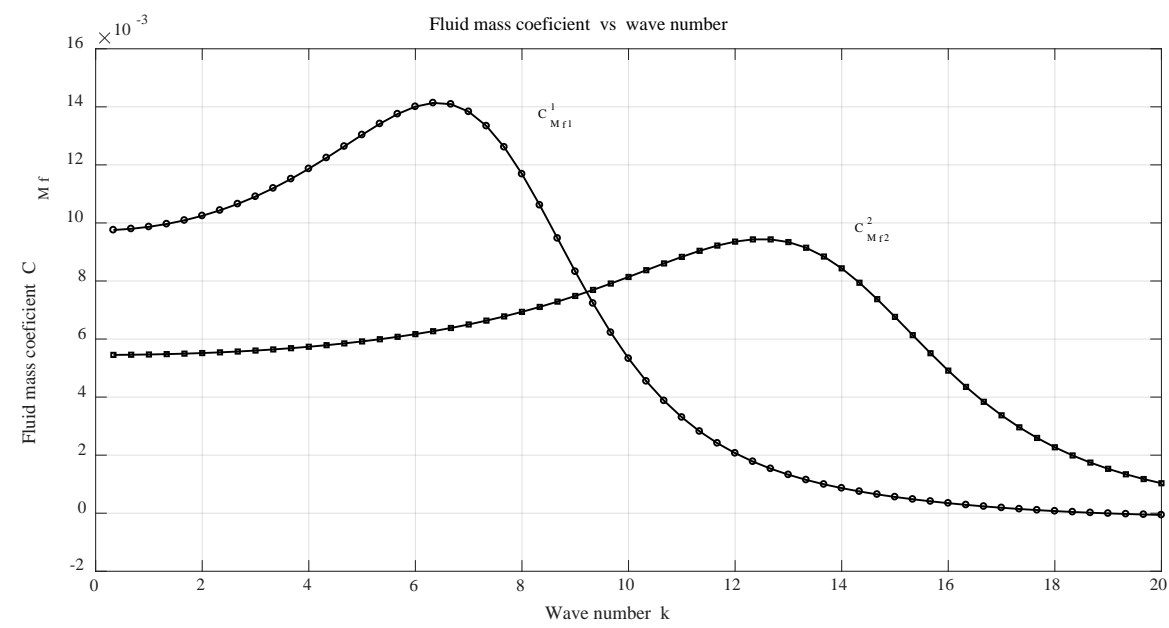

Fig.5. Fluid mass coefficient vs wave number $k$ for the rectangular membrane

Fig. 6 presents the frequency parameter $C_{\omega}$ as a function of the fluid density for various modes. A density variation has been taken from 0 to $10 \mathrm{~kg} / \mathrm{m}^{3}$. This parameter decreases with the fluid density, and the decrease rate is higher, the lower the values. Furthermore, in the same figure the frequency parameter of the membrane appears in vacuum (absence of fluid), which corresponds to the fluid density value of $\rho_{\infty}=0 \mathrm{~kg} / \mathrm{m}^{3}$.

Fig. 7 presents the variation of the damping ratio coefficient with the wave number $k=\frac{\omega}{a_{\infty}}$. This coefficient is defined as $\xi=-\frac{\left(M_{f}\right)_{\text {imag }} \cdot \omega}{C_{c r}}$ where $\left(M_{f}\right)_{\text {imag }}$ is the imaginary part of the diagonal term of the fluid mass matrix that contributes to the damping effect. $C_{c r}$ is the critical damping defined as $C_{c r}=2 \sqrt{K \cdot M_{T}}$ where $K$ and $M_{T}$ are the diagonal terms of the rigidity and total mass matrices. $\xi$ presents an oscillating variation with the wave number $k$, reaching a maximum for a certain value of $k$ and decreasing to a zero value for high values of $k$. This damping ratio due to acoustic losses is important for some values of the reduced frequency $k$, and must be taken into account because the value of the structural damping ratio for the material of the membrane can be of the order of 0.04 . 


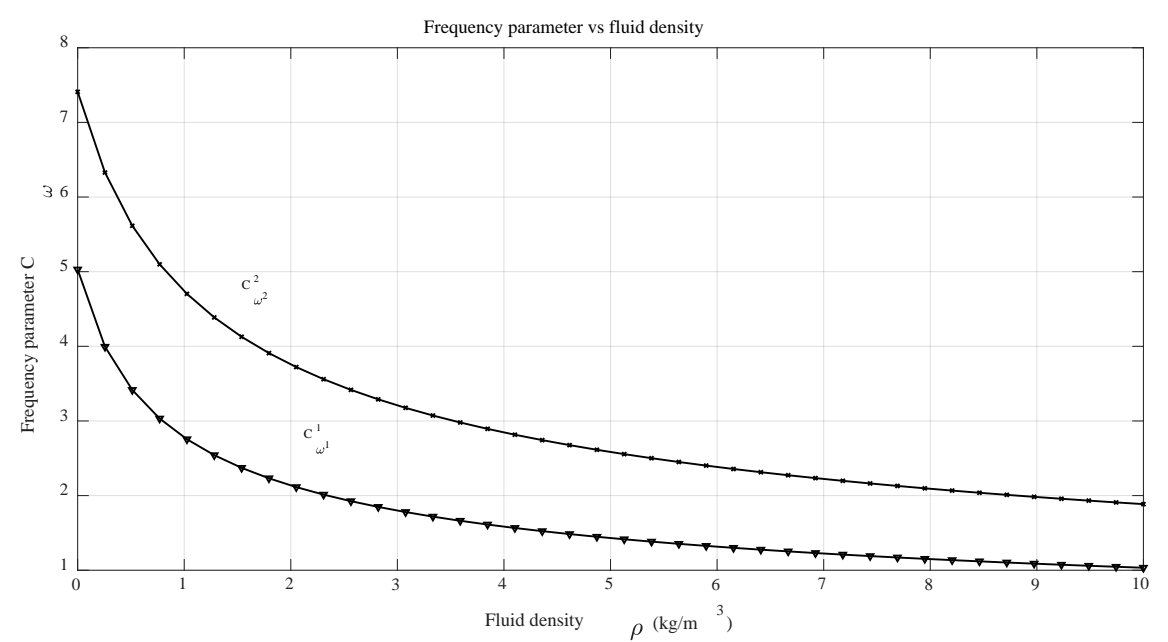

Fig. 6. Variation of membrane frequency parameter $C_{\omega}$ with fluid density for different nodal parameters

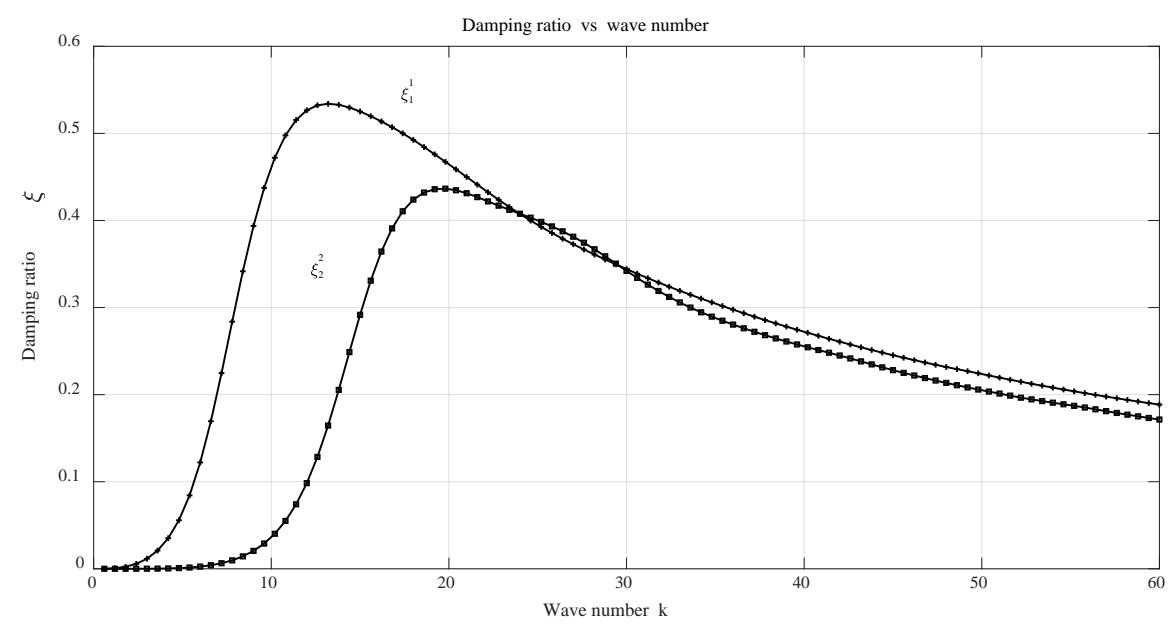

Fig.7. Damping ratio $\xi_{m}^{n}$ vs wave number $k$ for the rectangular membrane

Finally, in Table 2 the oscillation frequencies of the rectangular membrane submerged in fluid (air) for the different modes of the membrane are presented, and are compared concerning the values in the vacuum case. The properties of the rectangular membrane, as side dimensions, thickness, tension and material, are the above considered. As it is deduced, the reduction in the frequency, with the membrane submerged in air that is a light fluid, is quite appreciable with respect to the vacuum case (see Fowler et al. [1987]), and this reduction is higher the lower the value of the mode considered. These results are obtained for the case of air taking into account the compressibility effects, but they do not differ almost nothing in relation to the incompressible case. 
Table 2. Natural frequencies ( $\mathrm{Hz}$ ) for an aluminum rectangular membrane for the vacuum case and with air for different modes, with side lengths $L_{x}=50 \mathrm{~cm}$ and $L_{y}=40 \mathrm{~cm}$, and membranethickness $t_{h}=0.04 \mathrm{~mm}$

\begin{tabular}{|c|c|c|c|}
\hline$m, n$ & $f_{v}$ & $f_{a}$ & Frequency reduction (\%) \\
\hline 1,1 & 48.71 & 25.16 & 48.34 \\
\hline 1,2 & 71.77 & 43.32 & 39.64 \\
\hline 2,1 & 81.93 & 51.48 & 37.17 \\
\hline 2,2 & 97.42 & 64.72 & 33.57 \\
\hline
\end{tabular}

\section{Conclusions}

The problem of interaction of a structure with fluid has been studied for the case of a rectangularmembrane submerged in an infinite compressible fluid domain, as is the case with air. To accomplish that, a boundary element method has been employed and the acoust ic field pressure of the fluid is calculated in order to obtain the membrane frequencies of vibration. Results have been contrasted with those obtained by other authors and theories, showing thus the validity of this method. The effect of several parameters of the rectangular membrane on its vibroacoustic response has been analyzed, in particular the variation of the frequency parameter with the membrane dimensions, geometric aspect ratio and fluid density. Furthermore the variation of the fluid mass coefficient and the damping ratio with respect to the wave number has been obtained. Finally, results are presented for the rectangular membrane submerged in air and compared regarding the vacuum case.

\section{References}

Berry, A. et al [1990], “A general formulation for the sound radiation from rectangular, baffled plates with arbitrary boundary conditions”, The Journal of the Acoustical Society of America Vol. 88, 6

Caronti, A., Caliano, G., Iula, A. et al, [2002], “An Accurate Model for Capacitive Micromachined Ultrasonic Transducers”, IEEE Transactions on Ultrasonics, Ferroelectrics, and Frequency Control 49, 159-168.

Crighton, D.G. [1983], “The green function of an infinite fluid loaded membrane”, Journal of Sound and Vibration 86, 411-433

Crocker, M.J., [2007], Handbook of noise and vibration control, (John Wiley \& Sons, New Jersey).

Defay, E., Millon, C., Malhaire, C. et al, [2002], "PZT Thin Films Integration for the Realisation of a High Sensitivity Pressure Microsensor Based on a Vibrating Membrane”, Sensors and Actuators A 99, 64-67.

Erchiqui, F., Souli, M., Kanit, T. et al, [2015], "Characterization of polymeric membranes under large deformations using fluid-structure coupling”, Int. J. Applied Mechanics 7, (5).

Fahy, F., [2001], The foundations of engineering acoustics, (Elsevier Academic Press, London).

Fowler, J., Lagerquist, N., \& Leve, H. [1987], “Effect of air in modal tests”, Proceedings of the $5^{\text {th }}$ International Modal Analysis Conference, London.

Gascón-Pérez, M. \& García-Fogeda, P. [2015], "Induced damping on vibrating circular plates submerged in still fluid ”, Int. J. Applied Mechanics 7, (6).

Gascón-Pérez, M. [2017], “Acoustic influence on the vibration of a cylindrical membrane drum”, Int. J. Applied Mechanics 9, (5).

Grosjean, C.,Yang, X. \& Tai,Y. [1999], “A Practical Thermopneumatic Valve”, Proceedings of the 12th IEEE International Conference on Micro Electro Mechanical Systems, 147-152.

Jenkins, C.H.M. \& Korde, U.A. [2006], "Membrane vibration experiments: An historical review and recent results”, Journal of Sound and Vibration 295, 602-613

Jeong, O.C. \& Yang, S.S. [2000], "Fabrication and Test of a Thermopneumatic Micropump with a Corrugated p+ Diaphragm”, Sensors and Actuators 83, 249-255.

Kerboua, Y. [2008], "Vibration analysis of rectangular plates coupled with fluid”, Applied Mathematical Modelling 32, 2570-2586

Kwak, M.K. [1996], “Hydroelastic Vibration of Rectangular Plates”, Journal of Applied mechanics 63, 110115

Pan, L.S., Ng, T.Y., Wu, X.H. et al, [2003], “Analysis of Valveless Micropumps with Inertial Effects”, Journal of Micromechanics and Microengineering 13, 390-399.

Roussos, I.M., [2014], Improper Riemann integrals, (CRC Press, Boca Raton FL).

Wang, C.Y. \& Wang, C.M., [2014], Structural vibration: exact solutions for strings, membranes, beams and plates, (CRC Press, Boca Raton FL). 
Gascón-Pérez, M. [2018], "Interactions of an oscillating rectangular membrane with a compressible fluid”, Int. J. Applied Mechanics 10, (2). 